\title{
Discretized Diffusion Processes
}

\author{
Stefano Ciliberti, ${ }^{1}$ Guido Caldarelli, ${ }^{1}$ Paolo De Los Rios, ${ }^{2}$ Luciano Pietronero, ${ }^{1}$ and Yi-Cheng Zhang ${ }^{2}$ \\ ${ }^{1}$ INFM Sezione di ROMA1 Dipartimento Fisica, Università di Roma “La Sapienza,” Piazzale Aldo Moro 2, 00185 Roma, Italy \\ ${ }^{2}$ Institut de Physique Théorique, Université de Fribourg, CH-1700, Fribourg, Switzerland
}

(Received 31 May 2000)

\begin{abstract}
We study the properties of the "rigid Laplacian" operator; that is we consider solutions of the Laplacian equation in the presence of fixed truncation errors. The dynamics of convergence to the correct analytical solution displays the presence of a metastable set of numerical solutions, whose presence can be related to granularity. We provide some scaling analysis in order to determine the value of the exponents characterizing the process. We believe that this prototype model is also suitable to provide an explanation of the widespread presence of power law in a social and economic system where information and decision diffuse, with errors and delay from agent to agent.
\end{abstract}

PACS numbers: 05.40.-a, 64.60.Ak, 64.60.Fr, 87.10.+e

Most equations in science are continuous in value. While this is a good approximation to reality, natural processes often are discrete in microscopic details (e.g., atoms and molecules). We ask the following general questions: if the variables are subject to some small threshold such that no changes smaller than such a threshold are implemented, how do the continuous equations behave? Can we properly recover the continuous limit letting the threshold vanish? Are there new features emerging independent on the threshold value (and therefore robust down to the continuous limit)?

This problem is somewhat general and this is the reason for its importance. If we consider, in particular, the class of self-organized critical (SOC) systems then the question becomes crucial. We know that for many SOC systems, such as the Bak-Tang-Wiesenfeld [1] or the Zhang model [2], threshold and discretization play a dramatic role in order to avoid the diffusive behavior and to attain the critical one. By considering the continuous limit of the equations describing the microscopic dynamics of these cellular automata and by not preserving a finite threshold we get a trivial diffusive behavior instead of the critical one [3].

In order to investigate this kind of subject, we study here one of the oldest and most important equations of physics: the Laplace equation $\nabla^{2} \phi(x)=0$. Its time dependent version describes the diffusion of heat and of particles and the relaxation of incompressible, nonviscous fluids. In imaginary time it corresponds to the Schrödinger equation of quantum mechanics for a free particle (the difference vanishes completely if we are interested in stationary solutions/eigenstates). Therefore, we do not overestimate its importance if we consider it to be ubiquitous in physics. More recently, it was also considered to play a major role in fractal growth processes such as diffusion limited aggregation (DLA) [4] and the dielectric breakdown model (DBM) [5].

It is thus not a surprise that many methods have been developed to solve it, at least numerically. In general, both time and space are discretized on a lattice. The iterative method is the most commonly used method of solution, especially for problems such as DLA and DBM where the boundary conditions change in time. Essentially, the method resorts to iterating the equation

$$
\phi(i, t+1)=\frac{1}{2 d} \sum_{j . n . i} \phi(j, t),
$$

whose fixed point corresponds to the discretized version of $\nabla^{2} \phi(x)=0$. Numerically convergence to the fixed values $\phi(i)=\phi(i, t=\infty)$ has to be defined by some error: when two successive configurations differ by less than some small $\delta$ then the iteration stops. Of course $\delta$ has to be greater than the machine precision, $\delta^{\prime}$. This means that when the field $\phi$ is of the order of unity, it is impossible to change its value of quantities less than $\delta^{\prime}$.

Therefore it is of extreme importance to explore what happens if we explicitly set a precision in the definition of $\phi$, that is, if we say that we can change its value, according to (1), only by integer multiples of some chosen $\varepsilon$. This corresponds in considering the fields $\tilde{\phi}$

$$
\tilde{\phi}(i)=\operatorname{Int}_{\varepsilon}\left[\frac{1}{2 d} \sum_{j . n . i} \tilde{\phi}(j)\right] .
$$

$\operatorname{Int}_{\varepsilon}[x]$ corresponds to taking the integer multiple of $\varepsilon$ closest to $x$ (other possible definitions of the integer part, such as the lowest integer closest to $x$, have been explored and have been shown to give the same results). The relevance of this problem is not only related to the numerical solution of the Laplacian equation, but it comes also from real problems: granular materials, for example, behave sometimes as fluids. As such, we can expect them to obey some of the laws typical of fluids (hence also to relax according to the time dependent Laplacian equation), yet their intrinsic granularity forbids movements of quantities smaller than a single grain. In this respect we refer to the properties of $\tilde{\phi}$ as properties of rigid diffusion processes. Discrete-discrete Laplacian fields $\widetilde{\phi}$ (discrete in space-time, and discrete in the field $\tilde{\phi}$ ) can also play a role in modeling of economic and social systems. In particular, for the simplest case of the Laplacian "harmonic" operator the field is computed by averaging over the field neighbors. If this is viewed 
as a social system where opinions of players are formed by consulting people around, we would like to introduce a more realistic version, where decisions are considered only when a certain threshold of information is overcome and the diffusion is therefore discretized. This effect known as "rigidity" in the literature [3] is indeed responsible for the onset of criticality [6]. Since SOC is believed to play a role in both economic and social systems we suggest that our results can be viewed as a way to describe the appearance of criticality in such environments.

In this Letter we explore the behavior of this rigid Laplacian model (RLM). As we shall see, nontrivial properties emerge already in one dimension.

The standard setup of our simulations is the following: we take a $1 d$ lattice of $L+1$ sites, and we set the boundary conditions $\phi(0)=1$ and $\phi(L)=0$. Then we iterate the equation

$$
\phi(i, t+1)=\operatorname{Int}_{\varepsilon}\left[\frac{1}{2}[\phi(i-1, t)+\phi(i+1, t)]\right] .
$$

The iteration stops when there are no more possible rearrangements. The exact solution of $\nabla^{2} \phi=0$ on the lattice with the above boundary conditions is the straight line $\phi(i)=-i / L+1$. As we show in Fig. 1, the typical solution of (3) is instead a quadratic curve.

This can be easily explained by considering that as soon as a certain precision $\varepsilon$ is introduced, the Laplace equation can be considered as a first approximation as

$$
\nabla^{2} \phi=\alpha \varepsilon .
$$

In the case where the boundary conditions are $\phi(L)=0$ and $\phi(0)=1$ we obtain the following equation:

$$
\phi_{\varepsilon}(x)=\frac{1}{2} \alpha \varepsilon L^{2}\left(\frac{x}{L}\right)^{2}-\left(1+\frac{1}{2} \alpha \varepsilon L^{2}\right) \frac{x}{L}+1 .
$$

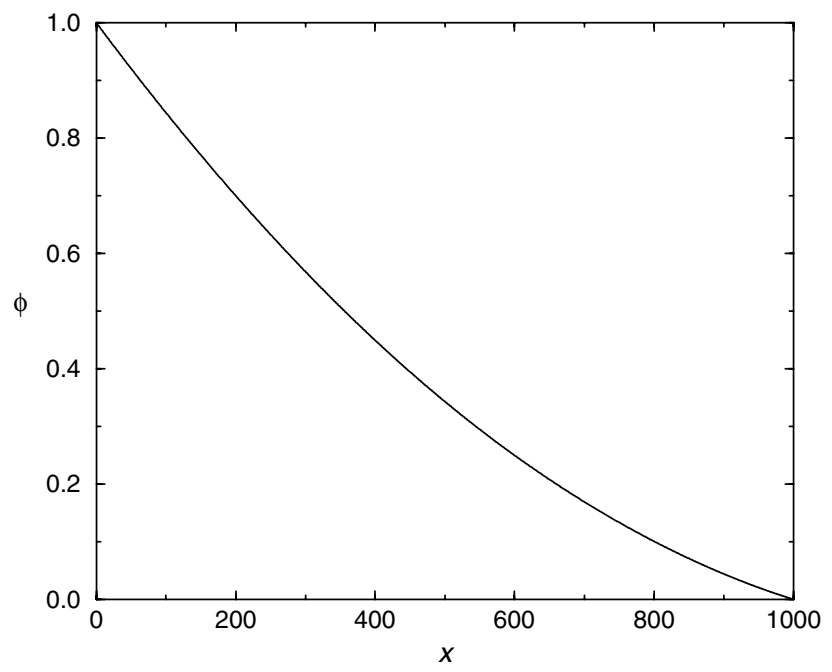

FIG. 1. Profile of $\phi$ for a $d=1$ system whose length is $L=$ 1000. At boundaries $\phi(0)=1, \phi(L)=0$.
In terms of the reduced variables $x^{\prime}=x / L$ and $\varepsilon^{\prime}=$ $\frac{1}{2} \alpha \varepsilon L^{2}$ one obtains

$$
\phi_{\varepsilon^{\prime}}\left(x^{\prime}\right)=\varepsilon^{\prime} x^{\prime 2}-\left(1+\varepsilon^{\prime}\right) x^{\prime}+1 .
$$

The precise value of $\alpha$ depends on the initial condition and it has to be determined with some fitting procedure. We can study the dynamics of the system observing that the evolution (1) always minimizes the functional

$$
E=\sum_{i=0}^{L-1}[\phi(i+1)-\phi(i)]^{2} .
$$

Equation (7) maps the RLM into the discrete space-time Gaussian model. Physically, since $\phi$ represents an electrostatic field, $E$ represents the total electrostatic energy of the sample, whose minimization in the case of fractal growth has been recently studied [7]. Letting the field $\phi$ take on only integer values leads to the discrete Gaussian model. The dynamics described by (3) is therefore a $T=0 \mathrm{dy}-$ namics, always decreasing the energy, and the stability of solutions different from the constant slope implies the presence of local energy minima. Since this process of energy minimization stops in local minima, we can perform some sort of simulated annealing by perturbing it. We increase the value of the field $\phi$ in some randomly chosen point by $n \varepsilon$, then we let the system relax. Indeed, we find that the curvature $\alpha$ decreases in time (time is defined in terms of perturbation steps; we consider the relaxation process to be much faster, as usual in self-organized critical models). Each perturbation implies a rearrangement of the profile.

For the energy of the system one has from Eq. (5)

$$
E \propto L^{3} \alpha^{2} \varepsilon^{2} .
$$

We know that for ordinary diffusion the energy variation $E$ is proportional to $t^{-3 / 2}$. We will show in the conclusion that based on that result, in this case one can expect $E \propto t^{1 / 2}$ or more precisely $E-E_{0} \propto\left(t / L^{z}\right)^{1 / 2}$, where we explicitly introduced a dynamical exponent $z$ for the process. Assuming for the moment this result, one has

$$
E \propto E_{0}-\left(t / L^{z}\right)^{1 / 2} \Rightarrow\left(t / L^{z}\right)^{1 / 2} \propto E_{0}-L^{3} \alpha^{2}\left(t / L^{z}\right) .
$$

By derivating this expression and by requiring that it shows no dependence on $L$ (unless for the ratio $t / L^{z}$ ) one obtains that the dynamical exponent must be $z=3$, in agreement with the value $z=3.0 \pm 0.1$ from numerical simulations, and

$$
\alpha(t, L)=\alpha_{0}-\left(t / L^{z}\right)^{1 / 2} f\left(t / L^{z}\right) .
$$

The scaling function $f(x)$ is such that $\alpha \rightarrow \alpha_{\infty} \neq 0$ for $t \gg L^{z}$. Such behavior has been tested in Fig. 2. We see that for this kind of system the relaxation process to the equilibrium is very slow because of granularity. Indeed, for a continuous Laplacian field the relaxation to the stationary state is an exponential process with a characteristic time scale $t_{0} \sim L^{2}$.

Such rearrangements proceed through power-law distributed avalanches, whose measure is the number of sites that changed their value of $\phi$. We show in Fig. 3 the 


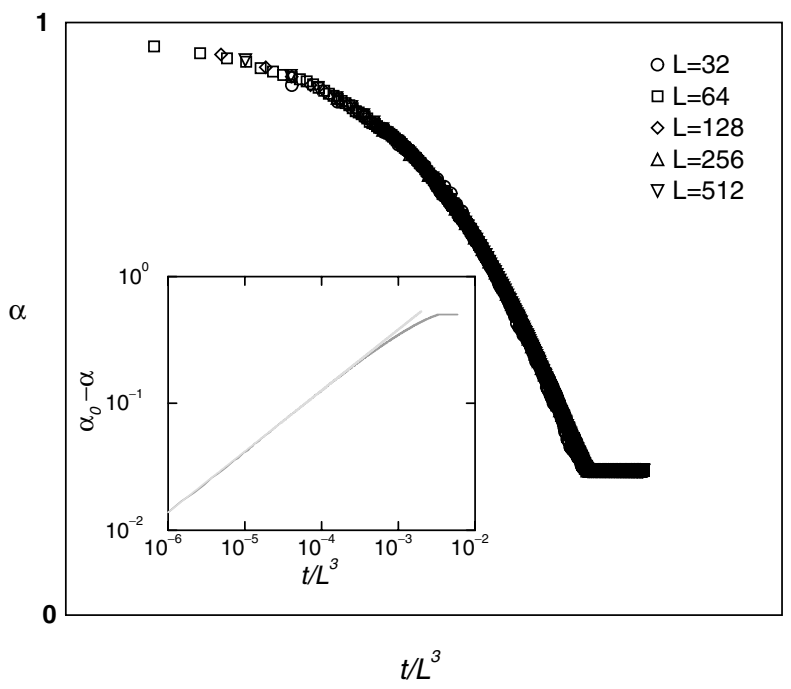

FIG. 2. Plot in rescaled time of $\alpha(t)$ and, in the inset, $\alpha(t)-\alpha_{0}$.

probability $p(s)$ to deal with an avalanche of size $s$. This function can be fitted with a power law of the kind $p(s) \propto$ $s^{-\tau}$. The value of the exponent $\tau$ measured from the data is $3.8 \pm 0.3$. To take into account the finite size effects of this distribution (the size of an avalanche cannot be greater than the size of the system) we can write the universal form $p(s, L)=s^{-\tau} g(s / L)$, where $g(x)$ is a scaling function such that $p(s=L)=0$. This scale invariant behavior describes the dynamics in a transient period where perturbations are accumulated into the system, until a limit profile (not necessarily the straight one) is reached. This transient state can be suitably delayed by considering a different dynamics where also negative perturbations are considered. To describe the evolution to the stationary state, we measured the average size $\langle s\rangle$ of the avalanches with respect to the time of the simulation.

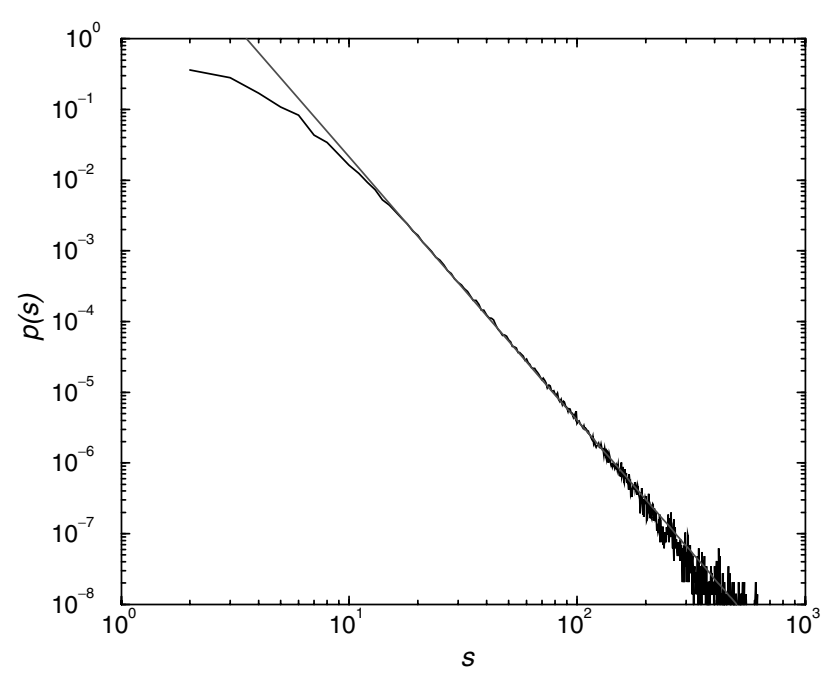

FIG. 3. Plot of the density function $p(s)$ giving the number of times that $s$ sites are updated in an avalanche.
In Fig. 4 we present the behavior of $\langle s(t)\rangle$ that also behaves as a power law with exponent $-1 / 3$. A simple scaling argument can be presented to explain such behavior. Indeed,

$$
\langle s(t, L)\rangle=L t^{-\nu} g\left(t / L^{z}\right),
$$

where we explicitly considered the finite size effects in the average size. In the above expression, $\nu$ represents the expected exponent and $z$ is the dynamical exponent previously introduced and present also in the scaling function $g$.

The collapse shown in the inset of Fig. 4 has been obtained with the following values: $z=3.0 \pm 0.1, \nu=$ $0.34 \pm 0.01$. The values of these two exponents are related; indeed the meaning of the dynamical exponent is as usual to determine the scale of time after which the process stops. By imposing that at the maximum time the average size of the avalanche is independent on $L$, one obtains the scaling relation

$$
1-z \nu=0
$$

from which $\nu=1 / z=1 / 3$ as numerically found in the limit $t \ll L^{3}$.

From this scaling relation it is possible to provide an argument to determine the value of the exponent $\tau$. Since the number of avalanches is the same whether they are classified according to their sizes [i.e., by means of the $p(s) d s$ ] or just counted in time (i.e., by means of $d t$ ) then one obtains $p(s)=\frac{d t}{d s}$. From the above scaling laws one finally obtains

$$
\tau=1+1 / \nu .
$$

We numerically tested the validity of these relationships as mentioned above. The rigidity of the field makes the process subdiffusive as implied by the value $z=3$ of the dynamical exponent, as opposed to the usual $z=2$ value. A more intriguing feature of these processes can be obtained by a suitable rescaling of the microscopic time scale.

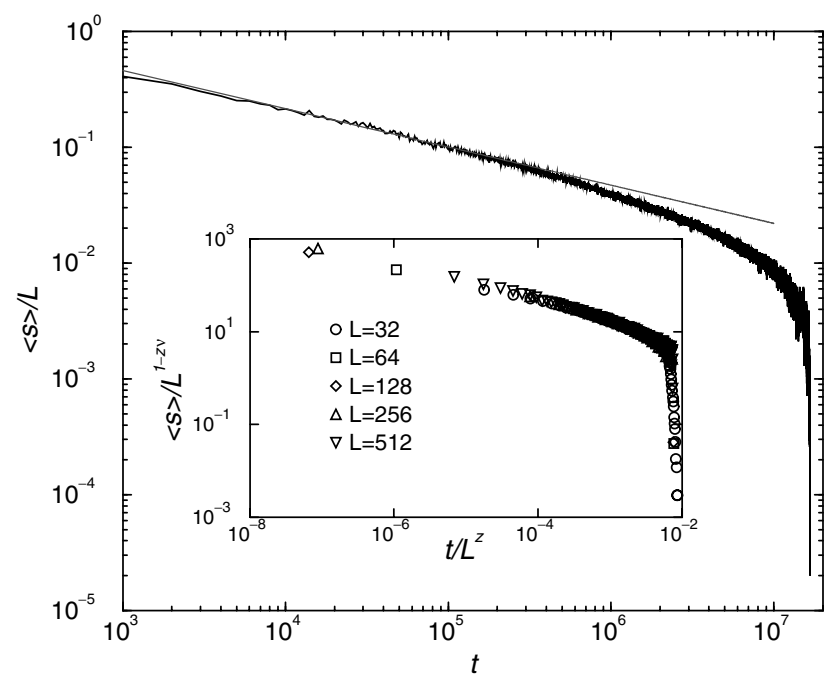

FIG. 4. $\langle s(t)\rangle$ versus $t$, in the inset collapse plot for different $L$ 's. 


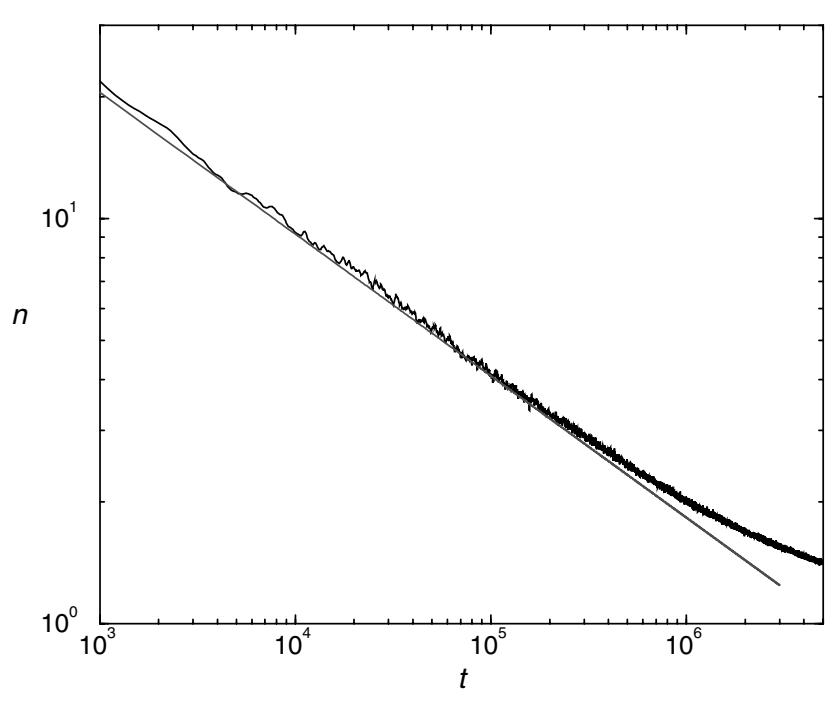

FIG. 5. Plot of $n(t)$; the smooth line is $y=t^{1 / 3}$.

As a matter of fact one can check the number $n(t)$ of microscopical sweeps needed in order to update the values of the field $\phi$ in an avalanche. This number $n(t)$ must be proportional to the average size of an avalanche at time $t$ and then it varies as $t^{-1 / 3}$, as can be shown by a direct measurement (Fig. 5). It means that the "physical" time of the process defined as $\sum_{t^{\prime}=1}^{t} n\left(t^{\prime}\right)$ behaves as $t^{2 / 3}$. By rescaling the scale of time with this characteristic time scale, the process loses any information on the granular nature of the medium and the dynamics behaves as a usual diffusive process. In this way we demonstrate the initial assumption for the energy $E$ in the system. In fact, since it varies for ordinary diffusion phenomena as $E \propto t^{\prime-3 / 2}$ and in this case the time of the process $t^{\prime}$ varies as $t^{\prime} \propto t^{-1 / 3}$, we obtain the above assumption of $E \propto t^{1 / 2}$.

We also checked the properties of this model in a dimension larger than $d=1$. We found a similar scale invariant behavior. In the case of $d=2$ we found a value of $\tau=2.65 \pm 0.03$, for $d=3$ we have $\tau=2.60 \pm 0.05$, and for $d=4$ we have $\tau=2.55 \pm 0.05$.

As described in the introduction, it would be tempting then to link this randomness to the known effect of rigidity in self-organized systems [3,6]. Self-organization has often been invoked as a way to describe the invariance observed in various social and economic systems [8-11]. Indeed we propose the basic mechanism of diffusion of money and/or information in a random environment (that can stop or reduce the information interchanged between players) as one of the reasons that could lead to scale invariance and to avalanche dynamics. In regards to the economic systems, we believe that this study could be also used to describe the time behavior of a quantized field with respect to the value of the truncation error. A specific example in this field is presented by considering the spurious effect in the price dynamics that could happen by chang- ing the tick at which they are traded (i.e., from $1 / 16$ of a dollar to $1 / 100$ ).

In conclusion, we introduced a model of diffusion, where the redistribution process is hindered by some sort of rigidity induced by the granularity of the fields. Randomness is present through a cutoff in the different possible values the field can assume. By truncating to a certain precision the value of the field a new and rich scenario of metastable states appears. The evolution of this system through the metastable states is driven by an avalanche dynamics with no particular time or length scale. By suitably rescaling the microscopic time of the evolution, one can use the scaling relations of the diffusive process to give a theoretical ansatz for the quantities describing the system. From computer simulations we find a good agreement between numerical data and theoretical ones. We believe this sort of process could be responsible for the ubiquitous presence of power-law relations in the everyday life. Clearly our work is readily generalized to other systems, notably Navier-Stokes, Kardar-Parisi-Zhang [12], or wave equations. One may wonder if there is some sort of superuniversality in their scaling behavior. Indeed a long list of similar problems awaits us to study.

P.D.L.R. and Y-C.Z. thank the Dipartimento di Fisica at the University of Rome "La Sapienza," for their kind hospitality. G. C. thanks for the same reason the Institut de Physique Théorique in Fribourg. This work has been supported by the European Network Contract No. FMRXCT980183.

[1] P. Bak, C. Tang, and K. Wiesenfeld, Phys. Rev. Lett. 59, 381 (1987).

[2] Y.-C. Zhang, Phys. Rev. Lett. 63, 470 (1989).

[3] R. Cafiero, V. Loreto, L. Pietronero, A. Vespignani, and S. Zapperi, Europhys. Lett. 29, 111 (1995).

[4] T. A. Witten and L. M. Sander, Phys. Rev. Lett. 47, 1400 (1981).

[5] L. Niemeyer, L. Pietronero, and H. J. Wiesmann, Phys. Rev. Lett. 52, 1033 (1984).

[6] A. Gabrielli, G. Caldarelli, and L. Pietronero, Phys. Rev. E (to be published).

[7] M. Marani, J. R. Banavar, G. Caldarelli, A. Maritan, and A. Rinaldo, J. Phys. A 31, L337-L343 (1998).

[8] G. K. Zipf, Human Behaviour and the Principle of Least Effort (Addison-Wesley, Cambridge, MA, 1949).

[9] B. B. Mandelbrot, J. Business 36, 394 (1963); R. N. Mantegna and H. E. Stanley, Nature (London) 376, 46 (1995).

[10] G. Caldarelli, M. Marsili, and Y.-C. Zhang, Europhys. Lett. 40, 479 (1997).

[11] G. Caldarelli, R. Marchetti, and L. Pietronero, Europhys. Lett. (to be published).

[12] M. Kardar, G. Parisi, and Y.-C. Zhang, Phys. Rev. Lett. 56, 889 (1986); C. Castellano, M. Marsili, and L. Pietronero, Phys. Rev. Lett. 80, 3527 (1998). 